\title{
Metabolic Imaging Allows Early Prediction of Response to Vandetanib
}

\author{
Martin A. Walter ${ }^{1,2}$, Matthias R. Benz ${ }^{2}$, Isabel J. Hildebrandt ${ }^{2}$, Rachel E. Laing ${ }^{2}$, Verena Hartung ${ }^{3}$, Robert D. Damoiseaux ${ }^{4}$, \\ Andreas Bockisch ${ }^{3}$, Michael E. Phelps ${ }^{2}$, Johannes Czernin ${ }^{2}$, and Wolfgang A. Weber ${ }^{2,5}$ \\ ${ }^{1}$ Institute of Nuclear Medicine, University Hospital, Bern, Switzerland; ${ }^{2}$ Department of Molecular and Medical Pharmacology, David \\ Geffen School of Medicine, UCLA, Los Angeles, California; ${ }^{3}$ Institute of Nuclear Medicine, University Hospital, Essen, Germany; \\ ${ }^{4}$ Molecular Shared Screening Resources, UCLA, Los Angeles, California; and ${ }^{5}$ Department of Nuclear Medicine, University Hospital, \\ Freiburg, Germany
}

The RET (rearranged-during-transfection protein) protooncogene triggers multiple intracellular signaling cascades regulating cell cycle progression and cellular metabolism. We therefore hypothesized that metabolic imaging could allow noninvasive detection of response to the RET inhibitor vandetanib in vivo. Methods: The effects of vandetanib treatment on the fullgenome expression and the metabolic profile were analyzed in the human medullary thyroid cancer cell line $T T$. In vitro, transcriptional changes of pathways regulating cell cycle progression and glucose, dopa, and thymidine metabolism were correlated to the results of cell cycle analysis and the uptake of ${ }^{3} \mathrm{H}$-deoxyglucose, ${ }^{3} \mathrm{H}-3,4$-dihydroxy-L-phenylalanine, and ${ }^{3} \mathrm{H}$-thymidine under vandetanib treatment. In vivo, the tumor metabolism under vandetanib was monitored by small-animal PET of tumor-bearing mice. Results: Vandetanib treatment resulted in the transcriptional downregulation of various effector pathways with consecutive downregulation of cyclin expression and $a G_{0} / G_{1}$ arrest. In vitro, vandetanib treatment resulted in the decreased expression of genes regulating glucose, 3,4-dihydroxy-L-phenylalanine, and thymidine metabolism, with a subsequent reduction in the functional activity of the corresponding pathways. In vivo, metabolic imaging with PET was able to assess changes in the tumoral glucose metabolism profile as early as $3 \mathrm{~d}$ after initiation of vandetanib treatment. Conclusion: We describe a metabolic imaging approach for the noninvasive detection of successful vandetanib treatment. Our results suggest that PET may be useful for identifying patients who respond to vandetanib early in the course of treatment.

Key Words: animal imaging; endocrinology; oncology; early treatment monitoring; medullary thyroid cancer; positron emission tomography; tyrosine kinase inhibitor; vandetanib

J Nucl Med 2011; 52:231-240

DOI: 10.2967/jnumed.110.081745

\footnotetext{
Received Jul. 27, 2010; revision accepted Nov. 3, 2010.

For correspondence or reprints contact: Martin A. Walter, Department of Molecular and Medical Pharmacology, David Geffen School of Medicine, 10833 Le Conte Ave., Los Angeles, CA 90095-6942.

E-mail: m.a.walter@gmx.net

Guest Editor: Norbert Avril, Queen Mary, University of London

COPYRIGHT @ 2011 by the Society of Nuclear Medicine, Inc.
}

$\mathbf{T}$ he RET (rearranged-during-transfection protein) protooncogene, located on chromosome 10q11.2, encodes for a tyrosine kinase of the cadherin superfamily that activates multiple intracellular signaling cascades regulating cell survival, differentiation, proliferation, migration, and chemotaxis (1). Gain-of-function mutations in the RET gene result in uncontrolled growth and cause human cancers and cancer syndromes, such as Hürthle cell cancer, sporadic papillary thyroid carcinoma, familial medullary thyroid carcinoma, and multiple endocrine neoplasia types $2 \mathrm{~A}$ and $2 \mathrm{~B}(2)$.

The signaling pathways downstream of RET are activated by autophosphorylation of several tyrosine and serine residues (Fig. 1A) and regulate several genes involved in tumor progression (3). Among other effects, activated Rac (Ras-related C3 botulinum toxin substrate) (4), STAT3 (signal transducer and activator of transcription 3) (5), Src (v-src sarcoma [Schmidt-Ruppin A-2] viral oncogene homolog) (5), Ras (v-Ha-ras Harvey rat sarcoma viral oncogene homolog) (6), and PI3K (phosphatidylinositol3-kinase) (7) each results in the upregulation of cyclin activity, which consequently leads to accelerated cell cycle progression (8). Growth factor receptor-bound proteins 7 and 10 (Grb7 and Grb10) presumably contribute to the invasive cell potential (9), whereas phospholipase C $\gamma$ (PLC $\gamma$ ) plays a central role in calcium signaling and cell survival (10).

With its signaling network leading to neoplastic transformation, the $R E T$ protooncogene is a highly attractive target for selective cancer therapy. Vandetanib (zd6474; Fig. $1 \mathrm{~B})$ is an orally bioavailable small-molecule inhibitor of the RET tyrosine kinase. It effectively inhibits the increased activity of ret as a consequence of RET/PTC3 mutations in papillary thyroid cancer and M918T RET mutations occurring in MEN2B-associated and sporadic medullary thyroid cancers (MTCs) (11). The pharmacokinetics of vandetanib allow once-daily oral administration, and grade 3 or 4 toxicity is found in about $33 \%$ of patients (12). The efficacy of vandetanib in medullary thyroid cancer is currently being evaluated in several phase II trials (13). These trials use 
FIGURE 1. (A) RET receptor and vandetanib. Single-pass transmembranous RET receptor plays central role in intracellular signaling cascades that regulate cellular survival, differentiation, proliferation, migration, and chemotaxis. (B) Structure of RET inhibitor vandetanib and network of $R E T$ mediated signaling events. RET autophosphorylation sites are shown with their direct targets. (C) In TT cell model, vandetanib displayed $\mathrm{IC}_{50}$ of $5.0 \mu \mathrm{M}$. (D) In untreated $\Pi$ cells, no expression of genes involved in Src and Shank3 pathways was present, whereas $3 \mathrm{~d}$ of treatment with vandetanib resulted in downregulation of genes involved mainly in STAT3 and Grb 7 and Grb10 pathways.

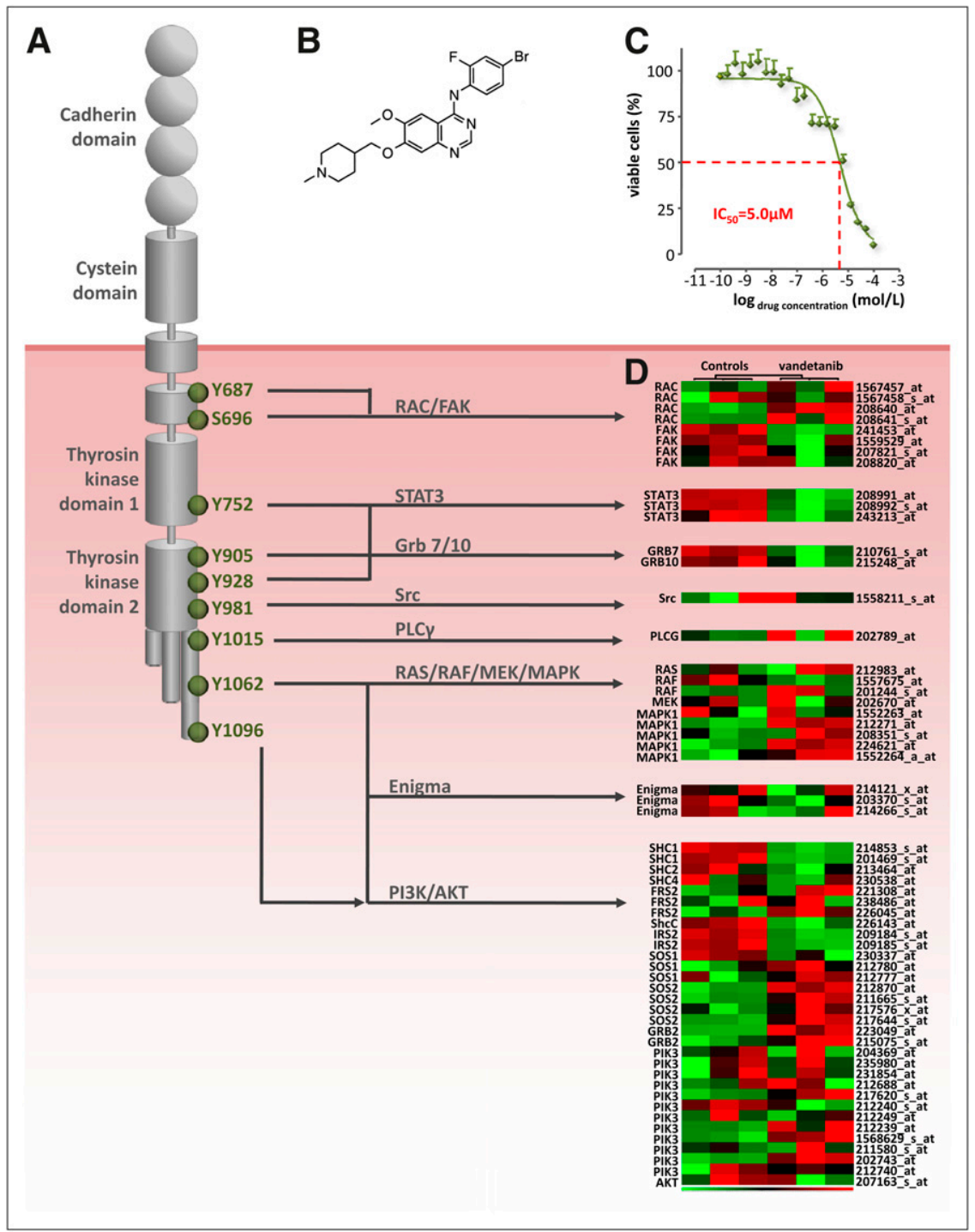

conventional tumor size criteria (World Health Organization or Response Evaluation Criteria in Solid Tumors (14)), which are limited in assessing responses because cytostatic drugs might not, or might only slowly, induce tumor shrinkage. Thus, there is a need for more sensitive readouts of successful RET kinase inhibition.

Accelerated metabolism of glucose is a characteristic feature of most cancer cells, including MTC, and is caused by increased expression activity of glucose transporters (GLUTs) as well as various glycolytic enzymes (15). Current literature data suggest that the activated RET protooncogene can modulate glucose metabolism via 5 pathways: PI3K (16) and PLC $\gamma$ (17) are physiologic mediators of insulin and increase glucose metabolism by upregulation of GLUT expression and hexokinase activity. Activated Src results in increased transcription of GLUT1 leading to an increase in cellular glucose uptake (18). Increased Ras GTPase expression leads to increased glucose transport, mainly because of increased GLUT transcription $(19,20)$. Finally, activation of STAT3 significantly induces GLUT expression and glucose uptake $(21,22)$. This possible relationship between $R E T$ signaling and tumor metabolic activity is of clinical interest because tumor glucose metabolism can be assessed quantitatively and noninvasively by PET with the glucose analog ${ }^{18} \mathrm{~F}$-FDG. Several studies have indicated that ${ }^{18} \mathrm{~F}-\mathrm{FDG}$ uptake is increased in subgroups of metastatic MTC and that ${ }^{18} \mathrm{~F}$-FDG PET can be used for restaging of patients with rising tumor markers. Using this clinical experience and the experimental data discussed, we hypothesized that ${ }^{18} \mathrm{~F}-\mathrm{FDG}$ PET may be used to monitor treatment with the RET inhibitor vandetanib in patients with MTC.

In addition to ${ }^{18}$ F-FDG PET, PET with radiolabeled 3,4dihydroxy-L-phenylalanine (DOPA) has been successfully used for staging of MTC. DOPA is taken up by the L-type amino acid transport system and is then metabolized to dopamine by DOPA decarboxylase. PET with radiolabeled 
DOPA may thus represent an alternative approach for imaging the biologic consequences of vandetanib treatment in MTC. Finally, mutated RET has been shown to trigger cell proliferation and increase cellular thymidine uptake (23). This process can be imaged clinically by PET with the thymidine analog fluorothymidine and may be another alternative for monitoring the effects of RET inhibition in MTC patients.

Thus, experimental and clinical data suggest that PET with radiolabeled ${ }^{18} \mathrm{~F}$-FDG, DOPA, or fluorothymidine may be used to assess response to vandetanib treatment in vivo. However, it is clear that glucose, DOPA, and thymidine metabolism are regulated by a complex network of several signaling pathways. To determine whether these metabolic processes are suitable for in vivo treatment monitoring, we systematically assessed the effects of vandetanib treatment on the transcription of transporters and enzymes involved in the metabolism of glucose, DOPA, and thymidine. We then studied the functional consequences of vandetanib treatment in vitro using radiolabeled ${ }^{18} \mathrm{~F}-\mathrm{FDG}$, DOPA, and fluorothymidine. On the basis of these data, we selected ${ }^{18} \mathrm{~F}-\mathrm{FDG}$ for further in vivo testing.

We report that vandetanib treatment downregulates cyclin activity with a consecutive $\mathrm{G}_{0} / \mathrm{G}_{1}$ arrest; that in vitro vandetanib treatment results in a decrease of glucose, DOPA, and thymidine metabolism; and that in vivo ${ }^{18} \mathrm{~F}$ FDG PET can assess response to vandetanib as early as $3 \mathrm{~d}$ after initiation. These results suggest that PET may be useful for identifying patients who respond to vandetanib early in the course of treatment.

\section{MATERIALS AND METHODS}

\section{In Vitro Model}

The human medullary thyroid cancer cell line TT (American Type Culture Collection) was established from an untreated 77-yold Caucasian woman. TT cells are a heterozygous carrier of a c634w (MEN2A-type) mutation in the cysteine-rich region of the extracellular domain of the RET gene. Cells were maintained in RPMI 1640 medium (Life Technologies, Inc.) supplemented with $16 \%$ fetal calf serum, penicillin $(100 \mathrm{IU} / \mathrm{mL})$, and streptomycin (10 $\mu \mathrm{g} / \mathrm{mL}$; Life Technologies, Inc.). Cells were grown at $37^{\circ} \mathrm{C}$ in an atmosphere of $5 \% \mathrm{CO}_{2}$.

\section{Viability Assay}

The antiproliferative activity of vandetanib was measured using the ATPlite luminescence assay (Perkin-Elmer). Cells were plated in 384-well plates $\left(3 \times 10^{3}\right.$ cells in $50 \mu \mathrm{L}$ of medium) using a Multidrop 384 (Thermo Fisher) and allowed to settle for $24 \mathrm{~h}$. Serial $2 \times$ drug dilutions were prepared with a final volume of 60 $\mu \mathrm{L}$ and final concentrations ranging from $10^{-4}$ to $10^{-10} \mathrm{M}$. The diluted compounds were added using a 500-nL custom 384-well pin tool (V\&P Scientific). Cells were incubated for $72 \mathrm{~h}$ at $37^{\circ} \mathrm{C}$, $5 \% \mathrm{CO}_{2}$, and then underwent an ATPlite assay to determine cell viability. The plates were measured on the Victor-3V using top read and an integration time of $0.5 \mathrm{~s}$. The results are presented as percentage of cell viability, compared with the dimethyl sulfoxide (DMSO) control. Inhibitory concentration of $50 \%\left(\mathrm{IC}_{50}\right)$ values were determined with curve-fitting analysis (nonlinear regression curve, sigmoidal dose-response), using an algorithm provided by Prism, version 5 (GraphPad Software).

\section{Cell Cycle Analyses}

Cells were plated in 6 -well plates $\left(1 \times 10^{5}\right.$ cells in $1 \mathrm{~mL}$ of medium). On the basis of the $\mathrm{IC}_{50}$ experiments, vandetanib at a final concentration of $5 \mu \mathrm{M}$ was added after $24 \mathrm{~h}$. For controls, vehicle (DMSO) without vandetanib was added. Cells were incubated at $37^{\circ} \mathrm{C}, 5 \% \mathrm{CO}_{2}$. At varying time points $(24,48$, and $72 \mathrm{~h}$ ), cells were trypsinized, washed, and resuspended in phosphatebuffered saline (PBS). Cell suspensions were stained with propidium iodide (Calbiochem) and RNase A (Sigma) in a hypotonic buffer. The DNA content was then analyzed by a FACScan flow cytometer (Becton Dickinson) using the CellQuest 3.1 software (Becton Dickinson) for acquisition and the ModFit LT 2.0 software (Verity) for analysis.

\section{Growth Curve Analyses}

Cells were plated in 6-well plates (Costar) at $1 \times 10^{5}$ per well. After $24 \mathrm{~h}$, vandetanib at a final concentration of $5 \mu \mathrm{M}$ was added. Control experiments were performed by adding vehicle (DMSO) without vandetanib. The number of viable cells was determined from triplicate wells after 24,48 , and $72 \mathrm{~h}$ by trypan blue exclusion using the Vi-Cell XR instrument (Beckman Coulter).

\section{Microscopy}

Serial TT images of the same cell populations with and without vandetanib treatment were obtained with an inverted microscope (TE2000S; Nikon), an automated $x, y, z$ stage (Applied Scientific Instrumentation Inc.), 2 automated shutters (Vincent Associates), and a charge-coupled device camera (CascadeII; Photometrics).

\section{RNA Extraction and Quality Control}

TT cell cultures were treated with $5 \mu \mathrm{M}$ vandetanib or vehicle in pentuplicate. After $72 \mathrm{~h}$ of treatment, cells were rinsed once with ice-cold PBS and lysed. Total RNA was isolated as previous described (24). Samples were analyzed on both a spectrophotometer and a 2100 Bioanalyzer (Agilent Technologies). For each condition, 3 samples with $28 \mathrm{~S} / 18 \mathrm{~S}$ ratios of more than 1.5 and no evidence of ribosomal peak degradation were included for DNA microarray hybridization.

\section{DNA Microarray Probe Preparation and Hybridization}

DNA microarray hybridization was performed in cooperation with the UCLA DNA Microarray Core. Total RNA purified from each sample was used to synthesize biotinylated complementary RNA (cRNA) for the Affymetrix GeneChip Array (Human Genome U133 Plus 2.0 oligonucleotide microarray). Therefore, $5 \mu \mathrm{g}$ of total RNA were used to start cDNA synthesis using polydT-24 primer containing a T7 RNA promoter. Biotin-labeled cRNA was synthesized using an Affymetrix RNA transcription labeling kit. The labeled cRNA was purified with RNeasy Mini Kit spin columns (Qiagen). The cRNA then was hybridized for $16 \mathrm{~h}$ with the Human Genome U133 Plus 2.0 array, which interrogates 38,500 well-characterized human genes (Affymetrix GeneChip Technology). After hybridization, the chips were washed and stained with R-phycoerythrin-streptavidin, and the signal was amplified with antistreptavidin and scanned with a GeneChip Scanner 3000 (Affymetrix). The intensity of each feature of the array was captured with an Affymetrix GeneChip Operating System, according to standard Affymetrix procedures. The messenger RNA abundance was determined using the average of the differ- 
ences between perfect match and intentional mismatch intensities for each probe set using the Affymetrix Microarray Suite 5.0 (MAS 5.0; Affymetrix) algorithm.

\section{Microarray Data Analysis}

The $c e l$ files generated by the GeneChip Operating System and DChip software (Harvard School of Public Health) were used to inspect all array images for defects and quality as well as for data summarization, normalization, filtering, and statistical analysis.

\section{Directed Data Analyses}

First, baseline expression and transcriptional changes under vandetanib treatment in the TT cell model were analyzed: in the main pathways downstream of RET (Rac/Ras-related C3 botulinum toxin substrate, STAT3, Grb7 and Grb10, Src, PLC $\gamma$, and Ras/Raf [V-raf-1 murine leukemia viral oncogene homolog 1]/ MEK [mitogen-activated protein kinase kinase 1]/MAPK [mitogen-activated protein kinase], Enigma, Shank3, PI3K/AKT [v-akt murine thymoma viral oncogene homolog 1]); in the main regulators of the cell cycle (cyclins, cyclin-dependent kinases (8)), and the relevant transporters and intracellular enzymes in glucose (class I GLUTs (25) and glycolysis pathway), DOPA (LAT1 and LAT2 of the SLC7 family of amino acid transporters (26): DDC [dopa decarboxylase], COMT [catechol- $O$-methyltransferase], and MAO [monoamine oxidase]), and thymidine metabolism (SLC29 family of nucleoside transporter family (27): thymidine kinase, deoxythymidylate kinase [DTYMK], and nucleosidediphosphate kinase $[\mathrm{NDPK}])$. For this analysis of a priori-defined transcripts, the $t$ test was used to assess significant changes $(P<$ 0.05) in expression under RET inhibition, whereas the Bonferroni adjustment was used in cases of multiple probes for 1 transcript.

\section{Undirected Data Analyses}

To identify further differentially expressed genes regulated by vandetanib treatment, the probe sets flagged absent were first filtered out. Differentially expressed probe sets were defined as those showing at least a 5-fold change in either direction with a statistically significant difference ( $t$ test, $P<0.05$ ), compared with the control group. All fold changes derived from the parametric model are based on means. To identify biologically relevant groups of genes affected by the treatment with vandetanib, we performed gene functional annotation and cluster analysis using the Database for Annotation, Visualization and Integrated Discovery Bioinformatics Resources 2008 from the National Institute of Allergy and Infectious Disease.

\section{Radiotracer Uptake Studies}

Glucose uptake was measured using $\left[1,2-{ }^{3} \mathrm{H}(N)\right]-2-d e o x y-D-$ glucose ( ${ }^{3} \mathrm{H}-2 \mathrm{DG}$; specific activity, $2.22 \mathrm{GBq} / \mathrm{mmol}$ [60 Ci/mmol]; Sigma Aldrich), DOPA uptake was measured using L-[ring2,5,6- $\left.{ }^{3} \mathrm{H}\right]-3$,4-dihydroxyphenylalanine $\left({ }^{3} \mathrm{H}\right.$-DOPA; specific activity, $2.22 \mathrm{GBq} / \mathrm{mmol}$ [ $60 \mathrm{Ci} / \mathrm{mmol}$ ]; Moravek Biochemicals), and thymidine uptake was assessed using [methyl- ${ }^{3} \mathrm{H}$ ] $3^{\prime}$-fluoro- $3^{\prime}$ deoxythymidine ( ${ }^{3} \mathrm{H}$-fluorothymidine; specific activity, 2.22 $\mathrm{GBq} / \mathrm{mmol}$ [60 Ci/mmol]; Moravek Biochemicals). Cells were plated in 6-well plates (Costar) at $1 \times 10^{5}$ per well. After $24 \mathrm{~h}$, vandetanib at a final concentration of $5 \mu \mathrm{M}$ was added for periods of 24,48 , and $72 \mathrm{~h}$. Control experiments were performed by adding vehicle without vandetanib. After different treatment periods, fresh cell culture medium containing ${ }^{3} \mathrm{H}-2 \mathrm{DG},{ }^{3} \mathrm{H}$-fluorothymidine, and ${ }^{3} \mathrm{H}$-DOPA at $37 \mathrm{kBq} / \mathrm{mL}(1 \mu \mathrm{Ci} / \mathrm{mL})$ were added to each well. Glucose-free medium was used for 2DG uptake studies. Cells were incubated at $37^{\circ} \mathrm{C}$ for $60 \mathrm{~min}$. Cells were then rinsed twice in icecold PBS and lysed with $0.1 \%$ sodium dodecyl sulfate. The cell lysates were collected, and scintillation fluid (ICN) was added to determine cell-associated ${ }^{3} \mathrm{H}$ radioactivity using a $\beta$-counter (5600; Packard). Radioactivity uptake values were normalized to the number of viable (trypan blue-negative) cells and expressed relative to untreated controls.

\section{In Vivo Model}

Severe combined immunodeficient (SCID/SCID) mice were purchased from The Jackson Laboratory. TT cells $\left(1 \times 10^{6} /\right.$ mouse $)$ growing exponentially in culture were resuspended in PBS and Matrigel (BD Biosciences) and injected subcutaneously at the right shoulder of the animal. Experiments were performed when the tumor diameter had reached more than $5 \mathrm{~mm}^{3}$ ( $\sim 4$ wk after tumor cell injection).

To assess the effect of vandetanib treatment on tumor growth, mice were paired according to the size of the TT cell xenograft and randomized into groups of 6 mice for intraperitoneal treatment either with vehicle or with vandetanib $(250 \mathrm{mg} / \mathrm{kg} / \mathrm{d}$ dissolved in PBS containing $0.5 \% \mathrm{v} / \mathrm{v}$ polysorbate 80 ). Tumor size was measured by calipers, and tumor volumes were calculated using the following equation: tumor volume $=0.5 \times$ (greatest diameter)

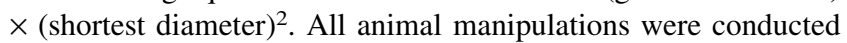
with sterile techniques following the guidelines of the University of California at Los Angeles Animal Research Committee.

\section{Histology and Mitotic Indices}

Tumors of mice randomized for treatment and vehicle were excised after $7 \mathrm{~d}$ of treatment and fixed in $4 \%$ buffered formalin, processed, and embedded in paraffin. The tissue-embedded paraffin blocks were cut, and slides were stained with Harris' hematoxylin and eosin. The mitotic index was based on the most mitotically active areas. Five fields were counted, and the mean number of mitotic figures per $40 \times$ magnification fields determined.

\section{In Vivo Small-Animal PET/CT}

Small-animal PET/CT scans were obtained in mice randomized for vandetanib treatment or vehicle using the microPET Focus 220 PET scanner (Siemens Preclinical Solutions) and MicroCAT II CT scanner (Siemens Preclinical Solutions). Mice were fasted for $12 \mathrm{~h}$ before ${ }^{18} \mathrm{~F}-\mathrm{FDG}$ injection and placed on a heating pad $\left(30^{\circ} \mathrm{C}\right)$ starting at $30 \mathrm{~min}$ before ${ }^{18} \mathrm{~F}$-FDG injection. For tracer injection and imaging, mice were anesthetized using 1.5\%-2\% isoflurane. Mice were imaged in a chamber that minimized positioning errors between PET and CT to less than $1 \mathrm{~mm}$. Imaging was started at $60 \mathrm{~min}$ after an intraperitoneal injection of $7.4 \mathrm{MBq}(200 \mu \mathrm{Ci})$ of ${ }^{18} \mathrm{~F}-\mathrm{FDG}$. Image acquisition time was $10 \mathrm{~min}$. Images were reconstructed by filtered backprojection, using a ramp filter with a cutoff frequency of 0.5 Nyquist. Image counts per pixel per second were calibrated to activity concentrations $(\mathrm{Bq} / \mathrm{mL})$ by measuring a $3.5-\mathrm{cm}$ cylinder phantom filled with a known concentration of ${ }^{18}$ F-FDG. Immediately after the PET scan, the mice underwent a 7-min micro-CT scan using routine image acquisition variables (70 kVp, $90 \mathrm{mAs}$, with 2-mm aluminum filters).

The AMIDE software was used to display PET and CT images and to define volumes of interest. The activity concentrations were expressed as percentage of the decay-corrected injected activity per gram of tissue. Spheric regions of interest $(2-\mathrm{mm}$ diameter) were placed on baseline and follow-up scans in the area of the tumor with the highest ${ }^{18} \mathrm{~F}$-FDG uptake. The mean standardized uptake value (SUV) was defined by the average 
pixel value within this volume of interest. Regions of interest were defined on fused PET/CT images generated by the AMIDE software to ensure reproducible positioning of the regions of interest.

\section{Statistical Analyses}

Discrete variables are summarized by counts and percentages, and continuous variables by their median and range, unless stated otherwise. Tumor sizes $(\mathrm{mm})$ and SUVs $(\mathrm{g} / \mathrm{mL})$ were compared using the Kruskal-Wallis 1-way ANOVA. To exclude any bias from varying tumor volumes in the in vivo model, we additionally used a binary regression model to test the correlation between the continuous variable SUV changes and the binary variable vandetanib treatment, with the volume changes as cofactor. All $P$ values were 2 -sided, and $P$ values of less than 0.05 were considered to be statistically significant. Data were analyzed using Statistica (version 6.0; Statsoft) software for Windows (Microsoft).

\section{RESULTS}

\section{Vandetanib Downregulates Various Effector Pathways with Consecutive $\mathbf{G}_{\mathbf{0}} / \mathbf{G}_{\mathbf{1}}$ Arrest}

Vandetanib displayed a sigmoidal dose-response curve in the ATPlite luminescence assay with an $\mathrm{IC}_{50}$ of $5.0 \mu \mathrm{M}$ (Fig. 1C). This concentration was subsequently used for all in vitro experiments, including the DNA microarray studies. In untreated TT cells, 26,338 of 54,613 probes $(48.2 \%)$ were present, including the transcripts for all major pathways driven by RET (Fig. 1D). Genome-wide screening revealed that vandetanib treatment resulted in significant transcriptional changes $(>5$-fold change, $P<0.05)$ in 103 genes, including 11 upregulated and 92 downregulated transcripts (Supplemental Fig. 1; supplemental materials are available online only at http://jnm.snmjournals.org). Functional annotations were available for 77 of these 92 downregulated transcripts, revealing the most significant changes in genes regulating cell cycle processes $(27 / 77$ genes, $35.1 \%, P=5.8 \mathrm{E}-15)$. Specifically, vandetanib treatment resulted in significant downregulation of transcripts including STAT3, Grb7, and Grb10 as well as of genes involved in the Ras/Raf/MEK/MAPK pathway (MEK and MAPK1) and the PI3K/AKT pathway (Src homology 2 domain containing transforming protein 1 , Src homology 2 domain containing transforming protein 2, Src homology 2 domain containing transforming protein 4, RAI1, IRS2, SOS1, SOS2, and GRB2; Fig. 1D).

Furthermore, vandetanib treatment resulted in significant downregulation of cyclins active in the $G_{1}$ phase (cyclin D1), during $G_{1}$ - to $S$-phase transition (cyclin E2), during the $S$ and $G_{2}$ phases (cyclin A1, cyclin A2), and during mitosis (cyclin B1, cyclin B2, Fig. 2A). In contrast, no transcriptional changes were found for any cyclin-dependent kinases. In separate experiments we examined the effect of vandetanib on the cell cycle by flow cytometry. Compared with vehicle, vandetanib treatment effectively reduced the S-phase fraction as early as $24 \mathrm{~h}$ after the start of treatment, with a correlating increase of the $G_{0} / G_{1}$ phase fraction. No increase in apoptosis was noted, suggesting cell cycle arrest in the $G_{0} / G_{1}$ phase before entering the $S$ phase (Fig. 2B). Proliferation of TT cell cultures was assessed over $72 \mathrm{~h}$ in the presence or absence of vandetanib. Untreated TT

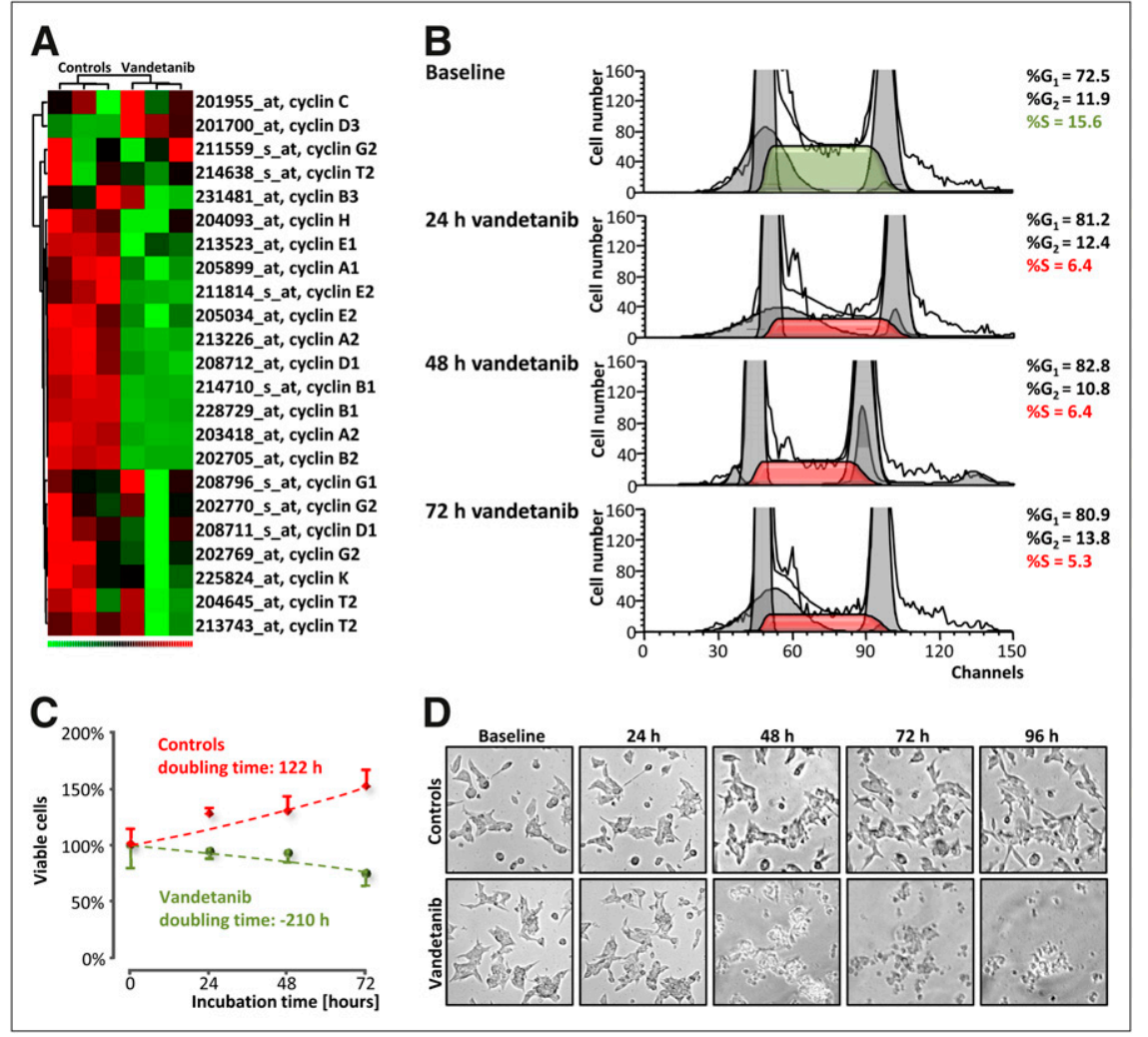

FIGURE 2. In vitro effects of vandetanib on cell cycle and cell growth. Treatment with vandetanib resulted in general downregulation of cyclins, including type $D, E$, and $A$ cyclins that regulate progression from $G_{1}$ to $S$ phase $(A)$. Accordingly, vandetanib induced arrest in G1 phase (B). Treatment effects of vandetanib on cell number (C) and morphology (D) in vitro are shown. 
cells displayed an exponential growth $\left(r^{2}=0.90\right)$, with a doubling time of $122 \mathrm{~h}$, whereas vandetanib induced a statistically significant treatment effect as early as $24 \mathrm{~h}$ after the start of treatment $(P=0.029$, Fig. 2C). Furthermore, serial microscopy revealed phenotypic treatment effects on morphology in all cells (Fig. 2D).

\section{Vandetanib Treatment Results in Decrease of Glucose, DOPA, and Thymidine Metabolism}

In untreated TT cells, only GLUT1 and GLUT3 of the class I GLUTs were expressed. In addition, transcripts were present also for class II (GLUT11) and class III (GLUT8 and GLUT12) GLUTs. Vandetanib treatment resulted in the transcriptional downregulation of class I GLUTs but not of any class II or class III GLUTs. Additionally, vandetanib treatment resulted in the downregulation of all genes involved in the glycolytic pathway, except glyceraldehyde-3-phosphate dehydrogenase, phosphoglycerate mutase 2 , and pyruvate dehydrogenase beta. In accordance with the transcriptional changes, the functional assay revealed a significant reduction of 2DG uptake to $37.4 \%$ of the baseline uptake after $3 \mathrm{~d}$ of vandetanib treatment ( $P=0.002$, Fig. $3 \mathrm{~A})$.

At baseline, the TT cells expressed 2 members of the solute carrier family 7: LAT1 (SLC7A5 and SLC7A7) and LAT2 (SLC7A6 and SLC7A8). Vandetanib treatment induced a downregulation of the transcripts for both transporters. In contrast, vandetanib treatment resulted in upregulation of transcripts for the intracellular enzymes of DOPA metabolism, DDC, COMT, and monoamine oxidase A. On the functional level, a reduction in DOPA uptake to $28.8 \%$ of baseline was found after $72 \mathrm{~h}$ of vandetanib treatment $(P=$ 0.012, Fig. 3B).

Transcripts for all members of the equilibrative nucleoside transporter family (SLC29A1, SLC29A2, SLC29A3, and SLC29A4) were present in the naïve TT cell line. After $72 \mathrm{~h}$ of vandetanib treatment, significant upregulation of SLC29A1, SLC29A2, and SLC29A3 was found. In contrast, vandetanib treatment was associated with downregulation of transcripts for the intracellular enzymes of thymidine metabolism: TK1, DTYMK, and NDPK. Parallel to the downregulation of these enzymes, a significant reduction in thymidine uptake to $28.7 \%$ of baseline appeared after $72 \mathrm{~h}$ of vandetanib treatment $(P=0.012$, Fig. $3 \mathrm{C})$

\section{Vandetanib Has Significant Antitumoral Effects in In Vivo TT Cell Xenotransplant Model}

TT cell xenografts in mice receiving only vehicle showed an exponential growth curve with a doubling time of $10.0 \mathrm{~d}$ ( $r^{2}=0.96$, Fig. 4A). In contrast, xenografts in mice randomized for vandetanib treatment showed a volume reduction to $50 \%$ within $9.0 \mathrm{~d}$. Significant treatment effects $(P=0.008)$ on the tumor volume were established after 10 $\mathrm{d}$ of vandetanib treatment. After withdrawal of vandetanib, all TT cell xenografts again displayed exponential growth $\left(r^{2}=0.94\right)$, with a mean doubling time of $5.1 \mathrm{~d}$.

In naïve TT cell xenografts, microscopy demonstrated few necrotic lagoons (magnification, $2 \times$ ) with a mitotic index of $15.0 \pm 6.4 / 40 \times$ field (magnification, $40 \times$ ). In contrast, after $14 \mathrm{~d}$ of vandetanib treatment, extensive necrotic lagoons were found along with a significant $(P=0.008)$ decrease of mitotic figures to $3.4 \pm 1.8 / 40 \times$ field (Fig. 3B).

FIGURE 3. In vitro effects of vandetanib on tumor cell metabolism. In vitro treatment with vandetanib resulted in downregulation of glucose carriers and glycolytic enzymes with consecutively reduced 2DG uptake (A), downregulation of glycoprotein-associated amino acid transporters with consecutively reduced DOPA uptake (B), and downregulation of enzymes of thymidine pathway with consecutively reduced thymidine uptake $(C)$ (representing mean value of 2 separate experiments conducted in triplicate). FLT = fluorothymidine.

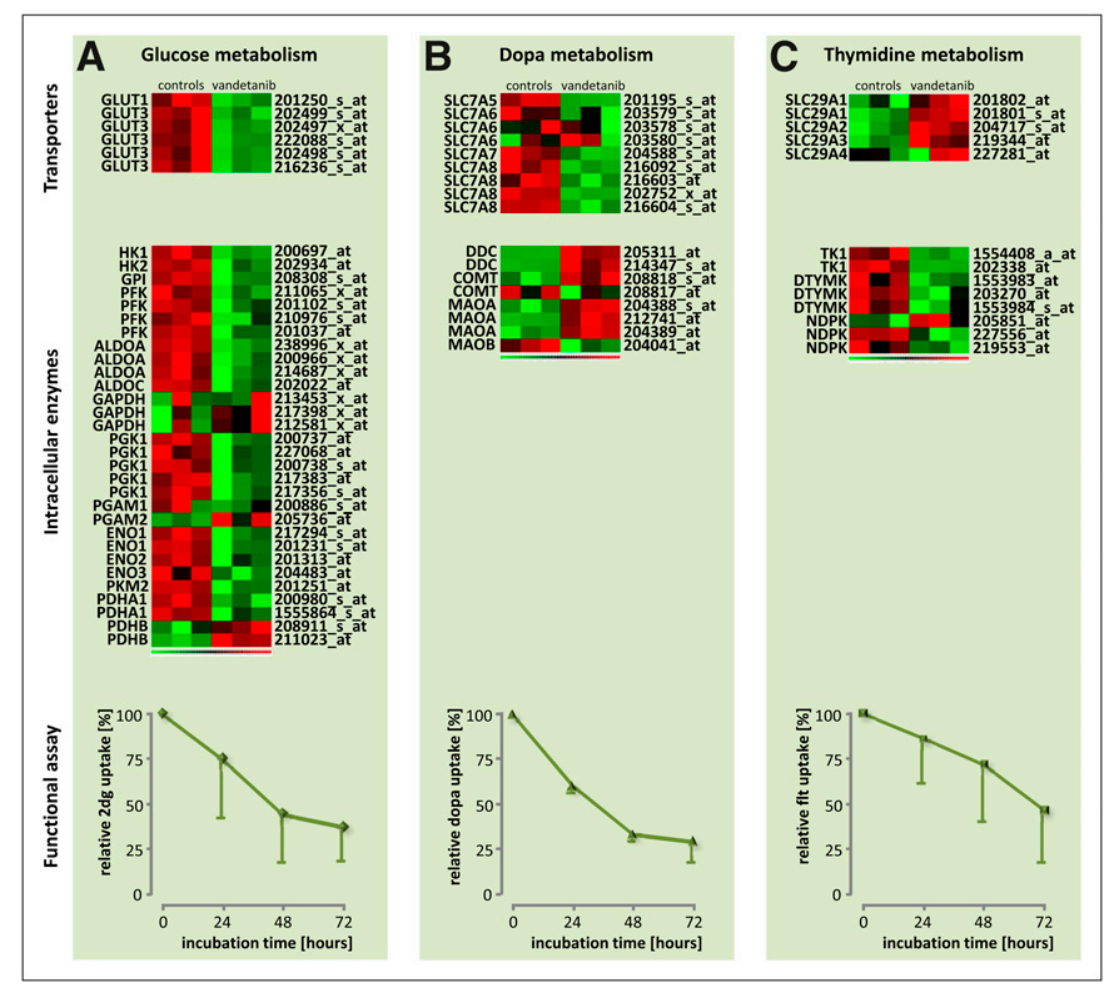




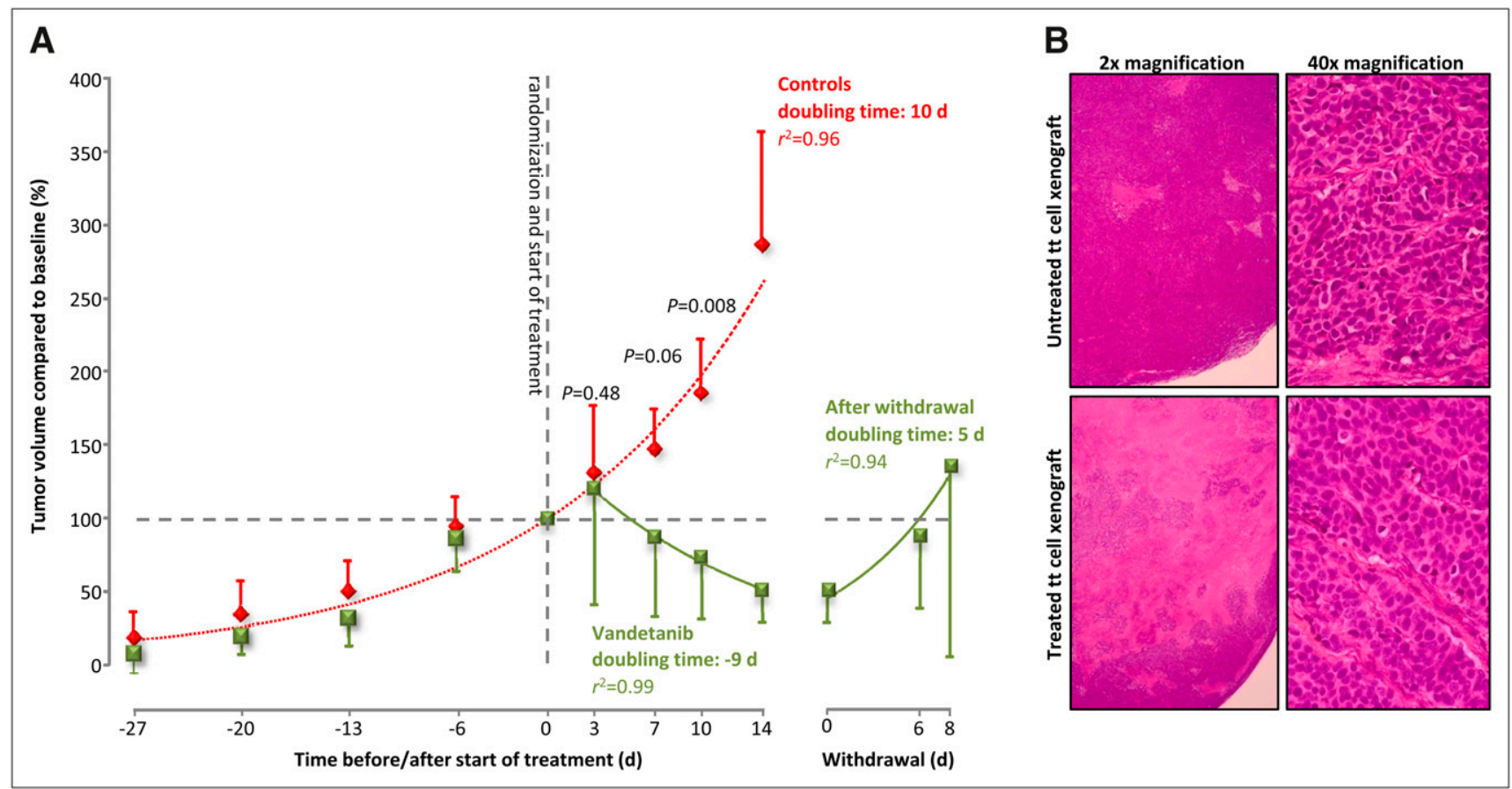

FIGURE 4. In vivo treatment model. Mice were paired and randomized to $1 \mathrm{mg}$ of vandetanib per day $(n=6)$ or no treatment $(n=6)$. Treatment with vandetanib resulted in significant reduction of tumor volume, whereas withdrawal resulted in recurrent tumor growth (A). In histologic sections, vandetanib-treated tumors showed extensive necrotic lacunae in comparison to untreated tumor tissues (magnification, $2 \times$ ). Additionally, under vandetanib therapy mitotic index dropped from $15 / 40 \times$ field to $4 / 40 \times$ field (B).

\section{Small-Animal PET Can Assess Response to Vandetanib Early in Course of Treatment}

Because the gene expression data indicated that of the 3 metabolic pathways studied vandetanib treatment had the most robust effects on genes involved in glycolysis, we used ${ }^{18} \mathrm{~F}$-FDG PET to monitor the effects of vandetanib in vivo. Mice bearing TT cell xenografts were imaged before and at 3, 7, and $14 \mathrm{~d}$ after the start of intraperitoneal administration of vandetanib or vehicle alone (Fig. 5A). Animals randomized to receive only vehicle displayed continually increasing tumoral ${ }^{18} \mathrm{~F}-\mathrm{FDG}$ uptake along with continually increasing tumor sizes. In contrast, in animals randomized for vandetanib, a continually decreasing tumoral ${ }^{18} \mathrm{~F}$-FDG uptake was found (Fig. 5B). The decrease in ${ }^{18} \mathrm{~F}-\mathrm{FDG}$ uptake was significant already after $3 \mathrm{~d}(P=0.002)$, whereas significant changes in tumor size $(P=0.008)$ were found only after $10 \mathrm{~d}$ of vandetanib treatment (Fig. 4A). After $14 \mathrm{~d}$ of vandetanib treatment, the tumoral ${ }^{18} \mathrm{~F}-\mathrm{FDG}$ uptake had decreased to $66.1 \% \pm 21.6 \%$, compared with the baseline values, and $30.9 \% \pm 10.1 \%$, compared with the uptake in the control animals. Additionally, the binary regression model confirmed the changes in ${ }^{18} \mathrm{~F}-\mathrm{FDG}$ uptake as factors independent from changes in tumor volume $(P<$ 0.001). This effect was reversible; after $7 \mathrm{~d}$ of discontinuation of vandetanib, the ${ }^{18} \mathrm{~F}-\mathrm{FDG}$ uptake increased to $88.9 \% \pm 25.1 \%$ of the baseline uptake.

\section{Clinical Case}

A 43-y-old man with biopsy-proven metastasized medullary thyroid cancer was treated with $300 \mathrm{mg}$ of vandetanib per day. In the week before as well as 12 and 24 wk after the start of treatment, he underwent standardized ${ }^{18} \mathrm{~F}-\mathrm{FDG}$ PET at the Nuclear Medicine Department, University Hospital Essen, Germany. Thereby, subsequent to fasting for at least $4 \mathrm{~h}, 300 \mathrm{MBq}$ of ${ }^{18} \mathrm{~F}-\mathrm{FDG}$ were intravenously applied, and whole-body scanning was conducted after $60 \mathrm{~min}$ on a full-ring bismuth germanate-based PET tomograph (Siemens Molecular Imaging). Glucose levels were within the reference range at each scanning time point $(84,92$, and 98 $\mathrm{mg} / \mathrm{dL}$ ). The maximum SUV was measured in 10 reference lesions at the baseline scan and at each follow-up scan. The SUV dropped from $8.1 \pm 1.5$ at baseline to $4.0 \pm 1.3$ after $12 \mathrm{wk}$ and $3.4 \pm 1.2$ after $24 \mathrm{wk}$ of treatment (Fig. 6).

\section{DISCUSSION}

In the studied TT cells, vandetanib treatment causes a $\mathrm{G}_{0} / \mathrm{G}_{1}$ arrest and the downregulation of key genes in the glycolysis pathway. As a result, vandetanib treatment results in a reduction of deoxyglucose uptake, both in vitro and in vivo. If these findings are confirmed in clinical studies, ${ }^{18} \mathrm{~F}$-FDG PET could be used to predict tumor response to vandetanib treatment early during the course of therapy.

\section{Cytostatic Effects of Vandetanib}

The rationale for selective targeting of RET is based on its key role for triggering cell cycle progression through its complex signaling network. In our medullary thyroid cancer in vitro model, vandetanib displayed an $\mathrm{IC}_{50}$ of $5.0 \mu \mathrm{M}$, which is within the range of patients' plasma concentra- 


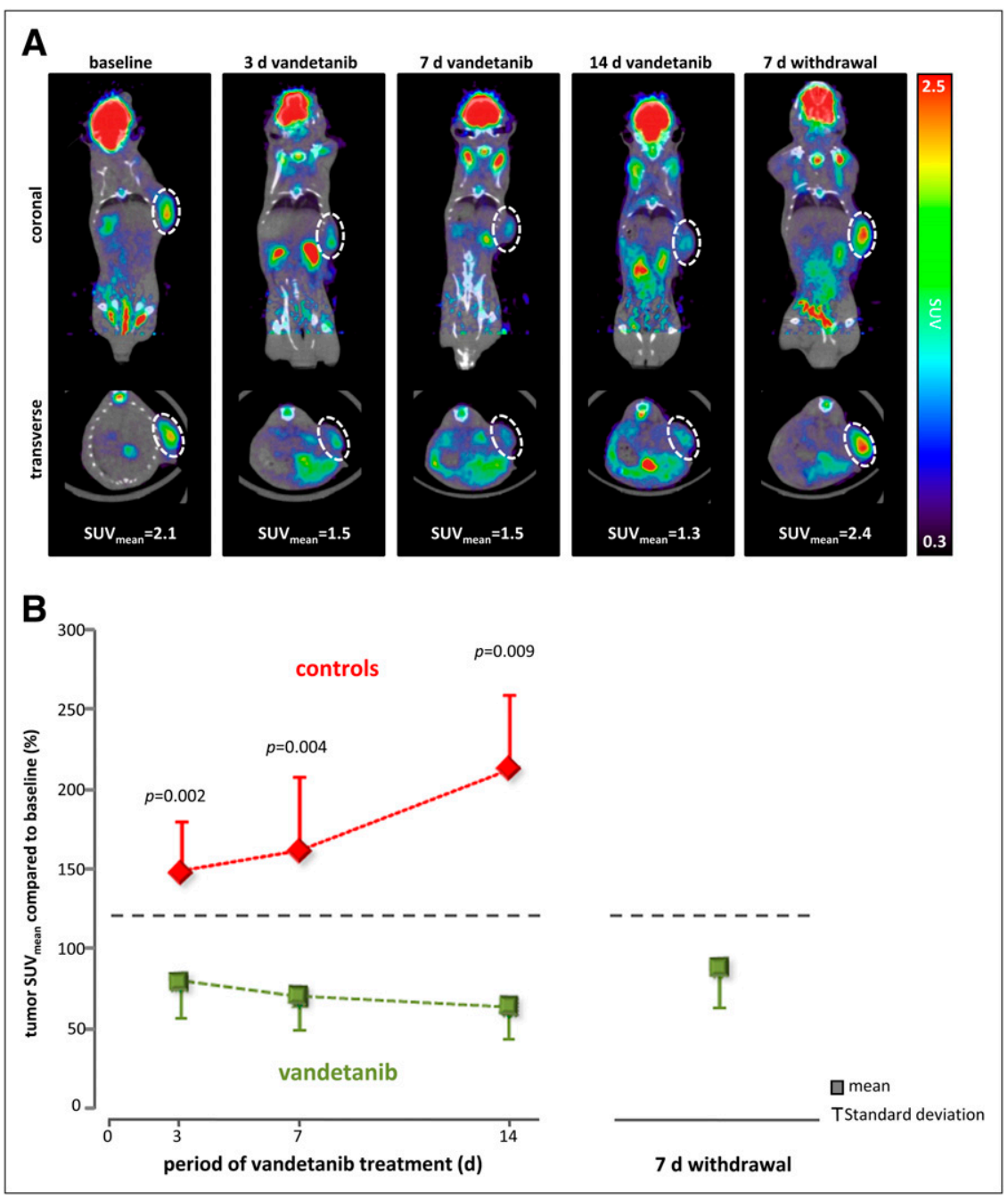

FIGURE 5. In vivo treatment monitoring. Metabolic changes under vandetanib treatment could be visualized using ${ }^{18} \mathrm{~F}$ small-animal PET (A). TT cell tumors of vandetanib-treated mice showed significantly lower ${ }^{18} \mathrm{~F}-\mathrm{FDG}$ uptake already at $3 \mathrm{~d}$ after initiation of treatment (B).

tions after oral administration (28). At this concentration, vandetanib treatment resulted in the transcriptional downregulation of various effector pathways in TT cells, especially the Rac, STAT3, and Grb7 and Grb10 pathways. Furthermore, interruption of RET signaling through the downstream pathways effectively resulted in the downregulation of cyclin expression and prevented cell proliferation.

\section{Metabolic Effects of Vandetanib}

Consistent with our hypothesis, vandetanib also significantly affected the metabolic profile after only $3 \mathrm{~d}$ of treatment. Gene expression profiling revealed transcriptional downregulation of key genes in glucose, DOPA, and thymidine metabolism. Among the RET-dependent pathways, transcriptional downregulation was mainly found in members of the Grb7 and Grb10 and STAT3 pathways. Grb10 has previously been implicated in the regulation of insulin receptor signaling (29). Overexpression of Grb10 in L6 myocytes and 3T3-L1 adipocytes has been shown to enhance insulin-induced glucose transport, glycogen synthesis, and lipogenesis, whereas expression of dominant- negative constructs, particularly the Grb10 SH2 domain, inhibited the insulin response (30). Interestingly, the Grb7/10/14 family was initially discovered through their binding to autophosphorylated epidermal growth factor receptors (EGFRs) (29), suggesting a potential role of the Grb7 and Grb10 pathways also in the modulation of ${ }^{18} \mathrm{~F}-$ FDG uptake found after EGFR inhibition (31). Similarly, also interleukin-induced activation of STAT3 has been shown to be essential for the expression of GLUTs, glucose uptake, and activation of the glycolytic pathway (21). Conversely, STAT3 inhibitors have been shown to block GLUT2 expression and 2DG uptake (22). Consistent with these observations, we found decreasing tumoral ${ }^{18} \mathrm{~F}-\mathrm{FDG}$ uptake after vandetanib-dependent downregulation of STAT3 and the Grb7 and Grb10 pathways.

In this study, the glycolytic pathway was the only metabolic pathway with downregulation of both transporters as well as intracellular enzymes. Furthermore, ${ }^{18}$ F-FDG has the best clinical availability and established value in medullary thyroid cancer imaging (32); therefore, it was selected for the in vivo imaging studies. In these in vivo studies, ${ }^{18} \mathrm{~F}-$ 
A

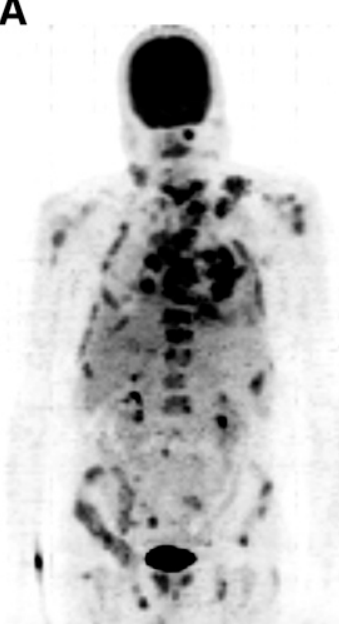

B

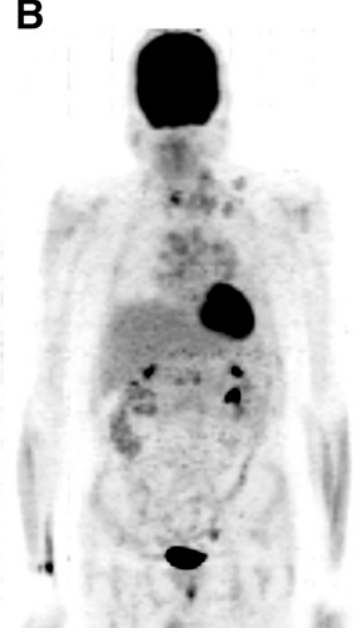

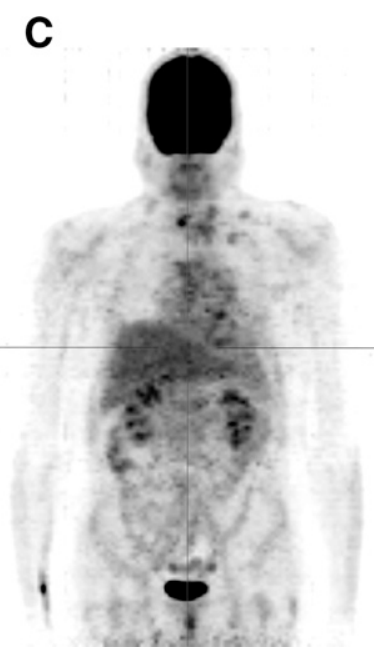

FIGURE 6. Clinical treatment monitoring. Serial ${ }^{18} \mathrm{~F}-\mathrm{FDG}$ PET scans of 43 -y-old man with metastasized medullary thyroid cancer before (A) as well as at 12 (B) and 24 (C) weeks after start of treatment with $300 \mathrm{mg}$ of vandetanib per day. Maximum SUV in 10 reference lesions dropped from $8.1 \pm 1.5$ at baseline to $4.0 \pm 1.3$ after $12 \mathrm{wk}$ and $3.4 \pm$ 1.2 after 24 wk of treatment.
FDG PET was able to reproduce the in vitro findings after only $3 \mathrm{~d}$ of vandetanib treatment. Importantly, the observed decrease in ${ }^{18} \mathrm{~F}$-FDG uptake after $3 \mathrm{~d}$ of treatment is not explained by cell death because there was no increase in apoptotic cells in vitro at this time and no decrease in tumor size in vivo. These results are consistent with clinical trials, in which tumor shrinkage in response to vandetanib has been rare, even when patient survival was significantly improved (12).

\section{Early Prediction of Treatment Response with Cytostatic Drugs}

The ability to predict tumor response early in the course of therapy offers the opportunity to intensify treatment in patients who are likely to respond to therapy. Conversely, treatment can be modified in patients who are unlikely to respond.

In a recent phase II clinical trial, the disease control rate, defined as complete response, partial response, or stable disease by morphologic CT criteria (Response Evaluation Criteria in Solid Tumors), was similar in the arm receiving vandetanib versus the control arm (57\% vs. $58 \%$ ). In contrast, the vandetanib arm had a significantly longer progression-free survival (hazard ratio, 0.76) (12). These results demonstrate the poor prognostic value of morphologic responses to vandetanib treatment.

${ }^{18}$ F-FDG PET has been validated as an early indicator of therapeutic responses in lymphoma (33) and a variety of solid tumors, such as gastrointestinal stromal tumors (GIST), soft-tissue sarcomas (34), ovarian cancer (35), small cell lung cancer (36), and adenocarcinoma of the oesophagogastric junction (37). In GIST tumors responding to the tyrosine kinase inhibitor imatinib (Gleevec), ${ }^{18} \mathrm{~F}$-FDG PET detects changes in tumor metabolism as early as $8 \mathrm{~d}$ after treatment initiation (38). Furthermore, ${ }^{18} \mathrm{~F}-\mathrm{FDG}$ PET predicts response as early as after 4 wk of therapy with the multitargeted tyrosine kinase inhibitor sunitinib in GIST (39). Vandetanib is a multitargeted tyrosine kinase inhibitor against EGFR, vascular endothelial growth factor receptor, and RET. RET activation is the main oncogenic event in medullary thyroid cancer (40). However, activity of other receptor tyrosine kinases, including vascular endothelial growth factor receptor and EGFR, are likely to contribute to tumor growth. Accordingly, ${ }^{18} \mathrm{~F}-\mathrm{FDG}$ PET represents readout of these combined effects of vandetanib treatment. The changes in the metabolic profile under vandetanib treatment found in this study provide a strong rationale to evaluate ${ }^{18} \mathrm{~F}$-FDG PET for the prediction of tumor response to RET kinase inhibitors in clinical trials.

\section{CONCLUSION}

A variety of novel approaches that target RET directly or indirectly have recently emerged, and an increasing number are currently being assessed in clinical trials (40). Additionally, a variety of alternative small-molecule inhibitors are currently being evaluated (13). With the increasing number of available treatment options, careful patient selection is necessary to ensure targeted therapy is administered to those most likely to gain clinical benefit. Therefore, the identification of surrogate markers of treatment efficacy is a key factor for the success of these novel treatment approaches. If validated in clinical trials, metabolic imaging may significantly affect selection and response readout of RET inhibition therapy.

\section{ACKNOWLEDGMENTS}

This study was supported in part by funds from the University Basel and the Swiss National Science Foundation.

\section{REFERENCES}

1. de Groot JW, Links TP, Plukker JT, Lips CJ, Hofstra RM. RET as a diagnostic and therapeutic target in sporadic and hereditary endocrine tumors. Endocr Rev. 2006;27:535-560.

2. Kouvaraki MA, Shapiro SE, Perrier ND, et al. RET proto-oncogene: a review and update of genotype-phenotype correlations in hereditary medullary thyroid cancer and associated endocrine tumors. Thyroid. 2005;15:531-544. 
3. Watanabe T, Ichihara M, Hashimoto M, et al. Characterization of gene expression induced by RET with MEN2A or MEN2B mutation. Am J Pathol. 2002;161:249-256.

4. Mettouchi A, Klein S, Guo W, et al. Integrin-specific activation of Rac controls progression through the $\mathrm{G}_{1}$ phase of the cell cycle. Mol Cell. 2001;8:115-127.

5. Sinibaldi D, Wharton W, Turkson J, Bowman T, Pledger WJ, Jove R. Induction of p21WAF1/CIP1 and cyclin D1 expression by the Src oncoprotein in mouse fibroblasts: role of activated STAT3 signaling. Oncogene. 2000;19:5419-5427.

6. Winston JT, Coats SR, Wang YZ, Pledger WJ. Regulation of the cell cycle machinery by oncogenic ras. Oncogene. 1996;12:127-134.

7. D'Amico M, Hulit J, Amanatullah DF, et al. The integrin-linked kinase regulates the cyclin D1 gene through glycogen synthase kinase 3beta and cAMP-responsive element-binding protein-dependent pathways. J Biol Chem. 2000;275: 32649-32657.

8. Malumbres M, Barbacid M. Cell cycle, CDKs and cancer: a changing paradigm. Nat Rev Cancer. 2009;9:153-166.

9. Pero SC, Daly RJ, Krag DN. Grb7-based molecular therapeutics in cancer. $E x$ pert Rev Mol Med. 2003;5:1-11.

10. Patterson RL, van Rossum DB, Nikolaidis N, Gill DL, Snyder SH. Phospholipase $\mathrm{C}$-gamma: diverse roles in receptor-mediated calcium signaling. Trends Biochem Sci. 2005;30:688-697.

11. Carlomagno F, Vitagliano D, Guida T, et al. ZD6474, an orally available inhibitor of KDR tyrosine kinase activity, efficiently blocks oncogenic RET kinases. Cancer Res. 2002;62:7284-7290.

12. Heymach JV, Paz-Ares L, De Braud F, et al. Randomized phase II study of vandetanib alone or with paclitaxel and carboplatin as first-line treatment for advanced non-small-cell lung cancer. J Clin Oncol. 2008;26:5407-5415.

13. Sherman SI. Early clinical studies of novel therapies for thyroid cancers. Endocrinol Metab Clin North Am. 2008;37:511-524.

14. Therasse P, Arbuck SG, Eisenhauer EA, et al. New guidelines to evaluate the response to treatment in solid tumors. European Organization for Research and Treatment of Cancer, National Cancer Institute of the United States, National Cancer Institute of Canada. J Natl Cancer Inst. 2000;92:205-216.

15. Macheda ML, Rogers S, Best JD. Molecular and cellular regulation of glucose transporter (GLUT) proteins in cancer. J Cell Physiol. 2005;202:654-662.

16. Samih N, Hovsepian S, Aouani A, Lombardo D, Fayet G. Glut-1 translocation in FRTL-5 thyroid cells: role of phosphatidylinositol 3-kinase and N-glycosylation. Endocrinology. 2000;141:4146-4155.

17. Wright DC, Craig BW, Fick CA, Lim KI. The effects of phospholipase C inhibition on insulin-stimulated glucose transport in skeletal muscle. Metabolism. 2002;51:271-273.

18. Salter DW, Baldwin SA, Lienhard GE, Weber MJ. Proteins antigenically related to the human erythrocyte glucose transporter in normal and Rous sarcoma virustransformed chicken embryo fibroblasts. Proc Natl Acad Sci USA. 1982;79: 1540-1544.

19. Hiraki Y, Rosen OM, Birnbaum MJ. Growth factors rapidly induce expression of the glucose transporter gene. J Biol Chem. 1988;263:13655-13662.

20. Rollins BJ, Morrison ED, Usher P, Flier JS. Platelet-derived growth factor regulates glucose transporter expression. J Biol Chem. 1988;263:16523-16526.

21. Guillet-Deniau I, Pichard AL, Kone A, et al. Glucose induces de novo lipogenesis in rat muscle satellite cells through a sterol-regulatory-element-binding-protein-1c-dependent pathway. J Cell Sci. 2004;117:1937-1944.

22. Suh HN, Lee YJ, Han HJ. Interleukin-6 promotes 2-deoxyglucose uptake through p44/42 MAPKs activation via $\mathrm{Ca} 2+/ \mathrm{PKC}$ and EGF receptor in primary cultured chicken hepatocytes. J Cell Physiol. 2009;218:643-652.
23. Hennige AM, Lammers R, Hoppner W, et al. Inhibition of Ret oncogene activity by the protein tyrosine phosphatase SHP1. Endocrinology. 2001;142: 4441-4447.

24. Walter MA, Seboek D, Demougin P, et al. Extraction of high-integrity RNA suitable for microarray gene expression analysis from long-term stored human thyroid tissues. Pathology. 2006;38:249-253.

25. Joost HG, Bell GI, Best JD, et al. Nomenclature of the GLUT/SLC2A family of sugar/polyol transport facilitators. Am J Physiol. 2002;282:E974-E976.

26. Verrey F, Closs EI, Wagner CA, Palacin M, Endou H, Kanai Y. CATs and HATs: the SLC7 family of amino acid transporters. Pflugers Arch. 2004; 447:532-542.

27. Baldwin SA, Beal PR, Yao SY, King AE, Cass CE, Young JD. The equilibrative nucleoside transporter family, SLC29. Pflugers Arch. 2004;447:735-743.

28. Kovacs MJ, Reece DE, Marcellus D, et al. A phase II study of ZD6474 (Zactima), a selective inhibitor of VEGFR and EGFR tyrosine kinase in patients with relapsed multiple myeloma: NCIC CTG IND.145. Invest New Drugs. 2006;24:529-535.

29. Holt LJ, Siddle K. Grb10 and Grb14: enigmatic regulators of insulin action-and more? Biochem J. 2005;388:393-406.

30. Deng Y, Bhattacharya S, Swamy OR, et al. Growth factor receptor-binding protein 10 (Grb10) as a partner of phosphatidylinositol 3-kinase in metabolic insulin action. J Biol Chem. 2003;278:39311-39322.

31. Su H, Bodenstein C, Dumont RA, et al. Monitoring tumor glucose utilization by positron emission tomography for the prediction of treatment response to epidermal growth factor receptor kinase inhibitors. Clin Cancer Res. 2006;12:5659-5667.

32. Khan N, Oriuchi N, Higuchi T, Endo K. Review of fluorine-18-2-fluoro-2deoxy-D-glucose positron emission tomography (FDG-PET) in the follow-up of medullary and anaplastic thyroid carcinomas. Cancer Control. 2005;12: 254-260.

33. Terasawa T, Nihashi T, Hotta T, Nagai H. ${ }^{18}$ F-FDG PET for posttherapy assessment of Hodgkin's disease and aggressive Non-Hodgkin's lymphoma: a systematic review. J Nucl Med. 2008;49:13-21.

34. Benz MR, Czernin J, Allen-Auerbach MS, et al. FDG-PET/CT imaging predicts histopathologic treatment responses after the initial cycle of neoadjuvant chemotherapy in high-grade soft-tissue sarcomas. Clin Cancer Res. 2009;15:28562863.

35. Avril N, Sassen S, Schmalfeldt B, et al. Prediction of response to neoadjuvant chemotherapy by sequential F-18-fluorodeoxyglucose positron emission tomography in patients with advanced-stage ovarian cancer. J Clin Oncol. 2005;23: 7445-7453.

36. Weber WA, Petersen V, Schmidt B, et al. Positron emission tomography in nonsmall-cell lung cancer: prediction of response to chemotherapy by quantitative assessment of glucose use. J Clin Oncol. 2003;21:2651-2657.

37. Lordick F, Ott K, Krause BJ, et al. PET to assess early metabolic response and to guide treatment of adenocarcinoma of the oesophagogastric junction: the MUNICON phase II trial. Lancet Oncol. 2007;8:797-805.

38. Stroobants S, Goeminne J, Seegers M, et al. ${ }^{18}$ FDG-Positron emission tomography for the early prediction of response in advanced soft tissue sarcoma treated with imatinib mesylate (Glivec). Eur J Cancer. 2003;39:2012-2020.

39. Prior JO, Montemurro M, Orcurto MV, et al. Early prediction of response to sunitinib after imatinib failure by ${ }^{18} \mathrm{~F}$-fluorodeoxyglucose positron emission tomography in patients with gastrointestinal stromal tumor. J Clin Oncol. 2009;27: 439-445.

40. Drosten M, Putzer BM. Mechanisms of disease: cancer targeting and the impact of oncogenic RET for medullary thyroid carcinoma therapy. Nat Clin Pract Oncol. 2006;3:564-74. 